NBER WORKING PAPER SERIES

\title{
WEATHER FORECASTING FOR WEATHER DERIVATIVES
}

\author{
Sean D. Campbell \\ Francis X. Diebold \\ Working Paper 10141 \\ http://www.nber.org/papers/w10141 \\ NATIONAL BUREAU OF ECONOMIC RESEARCH \\ 1050 Massachusetts Avenue \\ Cambridge, MA 02138 \\ December 2003
}

\begin{abstract}
We thank the editor, associate editor, and three referees for insightful comments that improved this paper, and we thank the Guggenheim Foundation, the National Science Foundation, the Wharton Financial Institutions Center, and the Wharton Risk Management and Decision Process Center for support. We are also grateful for comments by participants at the American Meteorological Society's 2001 Policy Forum on Weather, Climate and Energy, WeatherRisk 2002, and conferences at the Universities of Florence and Montreal, as well as Marshall Blume, Larry Brown, Geoff Considine, John Dutton, Rob Engle, John Galbraith, René Garcia, Stephen Jewson, Vince Kaminski, Paul Kleindorfer, Howard Kunreuther, Yu Li, Bob Livezey, Cliff Mass, Don McIsaac, Nour Meddahi, David Pozo, Matt Pritsker, S.T. Rao, Claudio Riberio, Til Schuermann, and Enrique Sentana. None of those thanked, of course, are responsible in any way for the outcome. The views expressed herein are those of the authors and not necessarily those of the National Bureau of Economic Research.
\end{abstract}

(C)2003 by Sean D. Campbell and Francis X. Diebold. All rights reserved. Short sections of text, not to exceed two paragraphs, may be quoted without explicit permission provided that full credit, including $(\mathrm{C}$ notice, is given to the source. 
Weather Forecasting for Weather Derivatives

Sean D. Campbell and Francis X. Diebold

NBER Working Paper No. 10141

December 2003

JEL No. G1

\section{$\underline{\text { ABSTRACT }}$}

We take a simple time-series approach to modeling and forecasting daily average temperature in U.S. cities, and we inquire systematically as to whether it may prove useful from the vantage point of participants in the weather derivatives market. The answer is, perhaps surprisingly, yes. Timeseries modeling reveals both strong conditional mean dynamics and conditional variance dynamics in daily average temperature, and it reveals sharp differences between the distribution of temperature and the distribution of temperature surprises. The approach can easily be used to produce not only short-horizon point forecasts, but also the long-horizon density forecasts of maximal relevance in weather derivatives contexts. We produce and evaluate both, with some success. We conclude that additional inquiry into nonstructural weather forecasting methods will likely prove useful in weather derivatives contexts.

Sean D. Campbell

Department of Economics

Brown University

Providence, RI 02912

Francis X. Diebold

Department of Economics

University of Pennsylvania

3718 Locust Walk

Philadelphia, PA 19104-6297

and NBER

fdiebold@wharton.upenn.edu 


\section{Introduction}

Weather derivatives are a fascinating new type of Arrow-Debreu security, making pre-specified payouts if pre-specified weather events occur. The market has grown rapidly. In 1997, the market for weather derivatives was nonexistent. In 1998 the market was estimated at \$500 million, but it was still illiquid, with large spreads and limited secondary market activity. More recently the market has grown to more than $\$ 5$ billion, with better liquidity. Outlets such as the Weather Risk (e.g., 1998, 2000) supplements to Risk Magazine and volumes such as Geman (1999) have chronicled the development.

Weather derivative instruments include weather swaps, vanilla options, option collars, and exotic (e.g., path-dependent) options. The underlying include heating degree days, cooling degree days, growing degree days, average temperature, maximum temperature, minimum temperature, precipitation (rainfall, snowfall), humidity and sunshine, among others - even the National Weather Service's temperature forecast for the coming week. Most trading is over-the-counter, but exchange-based trading is gaining momentum. Temperature-related weather derivatives, for example, are actively traded on the Chicago Mercantile Exchange (CME) for ten major U.S. cities.

A number of interesting considerations make weather derivatives different from "standard" derivatives. First, the underlying object (weather) is not traded in a spot market. Second, unlike financial derivatives, which are useful for price hedging but not quantity hedging, weather derivatives are useful for quantity hedging but not necessarily for price hedging (although the two are obviously related). That is, weather derivative products provide protection against weather-related changes in quantities, complementing extensive commodity price risk management tools already available through futures. Third, although liquidity in weather derivative markets has improved, it will likely never be as good as in traditional commodity markets, because weather is by its nature a location-specific and non-standardized commodity, unlike, say, a specific grade of crude oil.

Weather derivatives are also different from insurance. First, there is no need to file a claim or prove damages. Second, there is little moral hazard, although there is some, as when someone with a long 
precipitation position attempts to seed the clouds. (Don't laugh - it has happened!) Third, unlike insurance, weather derivatives allow one to hedge against comparatively good weather in other locations, which may be bad for local business (e.g., a bumper crop of California oranges may lower the prices received by Florida growers).

Weather forecasting is crucial to both the demand and supply sides of the weather derivatives market. Consider first the demand side: any firm exposed to weather risk either on the output (revenue) side or the input (cost) side is a candidate for productive use of weather derivatives. This includes obvious players such as energy companies, utilities and insurance companies, and less obvious players such as ski resorts, grain millers, cities facing snow-removal costs, consumers who want fixed heating and air conditioning bills, and firms seeking to avoid financial writedowns due to weather-driven poor performance. The mere fact that such agents face weather fluctuations, however, does not ensure a large hedging demand, because even very large weather fluctuations would create little weather risk if they were highly predictable. Weather risk, then, is about the unpredictable component of weather fluctuations "weather surprises," or "weather noise." To assess the potential for hedging against weather surprises, and to formulate the appropriate hedging strategies, one needs to determine how much weather noise exists for weather derivatives to eliminate, and that requires a weather model. What does weather noise look like over space and time? What are its conditional and unconditional distributions? Answering such questions requires statistical weather modeling and forecasting, the topic of this paper.

Now consider the supply side - sellers of weather derivatives who want to price them, arbitrageurs who want to exploit situations of apparent mispricing, etc. How should weather derivatives be priced? It seems clear that standard approaches to arbitrage-free pricing (e.g., Black-Scholes) are inapplicable in weather derivative contexts. In particular, there is in general no way to construct a portfolio of financial assets that replicates the payoff of a weather derivative. Hence the only way to price options reliably is by using forecasts of the underlying weather variable, in conjunction with a utility function, as argued for 
example by Davis (2001). This again raises the crucial issue of how to construct good weather forecasts (not only point forecasts, but also, and crucially, complete density forecasts), potentially at horizons much longer than those commonly emphasized by meteorologists. Hence the supply-side questions, as with the demand-side questions, are intimately related to weather modeling and forecasting.

Curiously, however, it seems that little thought has been given to the crucial question of how best to approach the weather modeling and forecasting that underlies weather derivative demand and supply. The meteorological weather forecasting literature focuses primarily on short-horizon point forecasts produced from structural physical models of atmospheric conditions (see, for example, the overview in Tribia, 1997). Although such an approach may well be best for helping one decide how warmly to dress today, it is not at all obvious that it is best for producing the long-horizon density forecasts relevant for weather derivatives. In particular, successful forecasting does not necessarily require a structural model, and in the last thirty years statisticians and econometricians have made great strides in using nonstructural models of time-series trend, seasonal, cyclical components to produce good forecasts, including longhorizon density forecasts. (For a broad overview see Diebold, 2004.)

In this paper, then, motivated by considerations related to the weather derivatives market, we take a nonstructural time-series approach to weather modeling and forecasting, systematically asking whether it proves useful. We are not the first to adopt a time-series approach, although the literature is sparse and inadequate for our purposes. The analyses of Harvey (1989), Hyndman and Grunwald (2000), Milionis and Davies (1994), Visser and Molenaar (1995), Jones (1996), and Pozo et al. (1998) suggest its value, for example, but they do not address the intra-year temperature forecasting relevant to our concerns. Seater (1993) studies long-run temperature trend, but little else. Cao and Wei (2001) and Torro, Meneu and Valor (2001) - each of which was written independently of the present paper - consider time-series models of average temperature, but their models are more restrictive and their analyses more limited.

We progress by providing insight into both conditional mean dynamics and conditional variance 
dynamics of daily average temperature as relevant for weather derivatives; strong conditional variance dynamics are a central part of the story. We also highlight the differences between the distributions of weather and weather surprises. Finally, we evaluate the performance of time series point and density forecasts as relevant for weather derivatives. The results are mixed but encouraging, and they point toward directions that may yield future forecasting improvements. We proceed as follows. In Section 2 we discuss our data and our focus on modeling and forecasting daily average temperature, and we report the results of time-series modeling. In section 3 we report the results of out-of-sample point and density forecasting exercises. In section 4 we offer concluding remarks and sketch directions for future research.

\section{Time Series Weather Data and Modeling}

We begin by discussing our choice of weather data and its collection. We are interested in daily average temperature $(T)$, which is widely reported and followed. Moreover, the heating degree days $(H D D \mathrm{~s})$ and cooling degree days $(C D D \mathrm{~s})$ on which weather derivatives are commonly written are simple transformations of daily average temperature. We directly model and forecast daily average temperature, measured in degrees Fahrenheit, for each of four measurement stations (Atlanta, Chicago, Las Vegas, Philadelphia) for 1/1/60 through 11/05/01, resulting in 15,285 observations per measurement station. Each of the cities is one of the ten for which temperature-related weather derivatives are traded at the CME. In an earlier and longer version of this article, Campbell and Diebold (2002), we report results for all ten cities, which are qualitatively identical. We obtained the data from Earth Satellite (EarthSat) corporation; they are precisely those used to settle temperature-related weather derivative products traded on the CME. The primary underlying data source is the National Climactic Data Center (NCDC), a division of the National Oceanographic and Atmospheric Administration. Each of the measurement stations supplies its data to the NCDC, and those data are in turn collected by EarthSat.

Before proceeding to detailed modeling and forecasting results, it is useful to get an overall feel for the daily average temperature data. In Figure 1 we plot the daily average temperature series for the last five 
years of the sample. The time-series plots reveal strong and unsurprising seasonality in average temperature: in each city, the daily average temperature moves repeatedly and regularly through periods of high temperature (summer) and low temperature (winter). Importantly, however, the seasonal fluctuations differ noticeably across cities both in terms of amplitude and detail of pattern.

In Figure 2 we show how the seasonality in daily average temperature manifests itself in unconditional temperature densities. The densities are either bimodal or nearly so, with peaks characterized by cool and warm temperatures. Also, with the exception of Las Vegas, each density is negatively skewed. The distributional results are in line with von Storch and Zwiers (1999), who note that although daily average temperature often appears Gaussian if studied over sufficiently long times in the troposphere, daily average surface temperatures may have different distributions, and with Neese (1994), who documents skewness and bimodality in daily maximum temperatures.

The discussion thus far suggests that a seasonal component will be important in any time-series model fit to daily average temperature, as average temperature displays pronounced seasonal variation, with both the amplitude and precise seasonal patterns differing noticeably across cities. We use a lowordered Fourier series to model this seasonality, the benefits of which are two-fold. First, it produces a smooth seasonal pattern, which accords with the basic intuition that the progression through different seasons is gradual rather than discontinuous. Second, it promotes parsimony, which enhances numerical stability in estimation. Such considerations are of relevance given the rather large size of our dataset (roughly 15,000 daily observations for each of four cities) and the numerical optimization that we subsequently perform.

One naturally suspects that non-seasonal factors may also be operative in the dynamics of daily average temperature. One such factor is trend, which may be relevant but is likely minor, given the short forty-year span of our data. We therefore simply allow for a simple low-ordered polynomial deterministic trend. Another such factor is cycle, by which we mean any sort of persistent covariance stationary 
dynamics apart from seasonality and trend. We capture cyclical dynamics using autoregressive lags.

The discussion thus far has focused on conditional mean dynamics, with contributions coming from trend, seasonal and cyclical components. We also allow for conditional variance (volatility) dynamics, with contributions coming from both seasonal and cyclical components. We approximate the seasonal volatility component using a Fourier series, and we approximate the cyclical volatility component using a GARCH process (Engle, 1982; Bollerslev, 1986). Assembling the various pieces, we estimate the following daily average temperature model for each of our four cities:

$$
T_{t}=\text { Trend }_{t}+\text { Seasonal }_{t}+\sum_{l=1}^{L} \rho_{t-l} T_{t-l}+\sigma_{t} \varepsilon_{t},
$$

where

$$
\begin{aligned}
& \text { Trend }_{t}=\sum_{m=0}^{M} \beta_{m} t^{m} \\
& \text { Seasonal } t=\sum_{p=1}^{P}\left(\delta_{c, p} \cos \left(2 \pi p \frac{d(t)}{365}\right)+\delta_{s, p} \sin \left(2 \pi p \frac{d(t)}{365}\right)\right) \\
& \sigma_{t}^{2}=\sum_{q=1}^{Q}\left(\gamma_{c, q} \cos \left(2 \pi q \frac{d(t)}{365}\right)+\gamma_{s, q} \sin \left(2 \pi q \frac{d(t)}{365}\right)\right)+\sum_{r=1}^{R} \alpha_{r} \varepsilon_{t-r}^{2}+\sum_{s=1}^{s} \beta_{s} \sigma_{t-s}^{2} \text { (1c) } \\
& \varepsilon_{t} \sim \operatorname{iid}(0,1),
\end{aligned}
$$

and $d(t)$ is a repeating step function that cycles through $1, \ldots, 365$. In all that follows we set $L=25, M=1$, $P=3, Q=3, R=1$, and $S=1$, which both Akaike and Schwarz information criteria indicate are more than adequate for each city. Maintaining the rather large value of $L=25$ costs little given the large number of available degrees of freedom, and it helps to capture long-memory dynamics, if present, as suggested by results such as those of Bloomfield (1992). Following Bollerslev and Wooldridge (1992), we consistently estimate this regression model with GARCH disturbances by Gaussian quasi maximum likelihood.

Now let us discuss the estimation results. First, and perhaps surprisingly, most cities display a statistically significant trend in daily average temperature. In most cases, the trend is much larger than the increase in average global temperature over the same period. For example, the results indicate that the daily average temperature in Atlanta has increased by three degrees in the last forty years. Such large trend increases are likely a consequence of development and air pollution that increased urban temperatures in 
general, and urban airport temperatures in particular, where most of the U.S. recording stations are located, a phenomenon often dubbed the "heat island effect." Second, the conditional mean seasonality is of course statistically significant and crucially important. We plot the estimated Fourier seasonal pattern in Figure 3. Third, daily average temperature displays strong cyclical persistence. The estimated autoregressions display an interesting root pattern, common across all four cities, regardless of location. The dominant root is large and real, around 0.85 , the second and third roots are a conjugate pair with moderate modulus, around .3 , and all subsequent roots are much smaller in modulus.

In Figure 4 we show model residuals over the last five years of the estimation sample. The fit is typically very good, with $R^{2}$ above $90 \%$. Figure 4 also provides a first glimpse of an important phenomenon: pronounced and persistent time-series heteroskedasticity in the residuals. In particular, weather risk, as measured by its innovation variance, appears seasonal, as the amplitude of the residual fluctuations widens and narrows over the course of a year. It seems that such seasonal heteroskedasticity in temperature was first noted, informally, in an economic context by Roll (1984). Subsequently in this paper we propose quantitative models and forecasts that explicitly incorporate the heteroskedasticity.

In Figure 5 we show residual kernel density estimates for each of the models. Four features stand out. First, average temperature residuals are much less variable than average temperature itself; that is, weather surprises are much less variable than the weather itself, with residual standard deviations only one third or so of the average temperature standard deviations. Second, the spreads of the residual densities vary noticeably across cities, indicating that weather risk is much greater in some cities than in others. Third, all of the residual densities are uni- as opposed to bi-modal, in contrast to the unconditional densities examined earlier, due to the model's success in capturing seasonal highs and lows. Fourth, all of the residual densities have only moderate negative skewness and moderate excess kurtosis; the average residual skewness and kurtosis coefficients are -0.36 and 4.10 .

In Figures 6 and 7 we display the correlograms of the residuals and squared residuals, taken to a 
maximum displacement of 800 days. The residual autocorrelations are negligible and appear consistent with white noise, indicating that we have modeled linear dependence adequately. Figure 7, however, reveals strong nonlinear residual dependence, driven by strong conditional variance dynamics.

In Figure 8 we plot the estimated residual conditional standard deviation from 1996 through 2001. The basic pattern is one of strong seasonal volatility variation, with upward-brushing GARCH effects, the persistence of which varies across cities. For each city, seasonal volatility appears highest during the winter months. Among other things, this indicates that correct pricing of weather derivatives may in general be crucially dependent on the season covered by the contract. Some cities display a great deal of seasonal volatility variation - the conditional standard deviation of Atlanta temperature shocks, for example, roughly triples each winter - whereas temperature shock volatility in other cities such as Las Vegas varies less across seasons.

It is interesting to note that the upward-brushing GARCH volatility effects typically occur in winter, when the volatility seasonal component is high, which might suggest the desirability of a multiplicative volatility specification. Nelson's (1991) exponential GARCH(1,1) is one attractive such specification, replacing the volatility specification for $\sigma_{t}^{2}$ in equation (1c) with an alternative but related specification for $\ln \sigma_{t}^{2}$. Estimation of exponential GARCH models, however, produced fitted conditional variance series nearly identical to those of the original GARCH models.

In Figure 9 we show density estimates for the standardized residuals, $\left(T_{t}-\hat{T}_{t}\right) / \hat{\sigma}_{t}$. The standardized residual densities still displays negative skewness; the average across cities is -0.45 . Modeling the conditional heteroskedasticity does, however, reduce (but not completely eliminate) residual excess kurtosis; the average across cities is now 3.74. Finally, in Figure 10 we show the correlogram of squared standardized residuals; there is no significant deviation from white noise behavior, indicating that the fitted model (1) is adequate.

\section{Time-Series Weather Forecasting}


Armed with a hopefully adequate nonstructural time-series model for daily average temperature, we now proceed to examine its performance in out-of-sample weather forecasting. We begin by examining its performance in short-horizon point forecasting, despite the fact that short horizons and point forecasts are not of maximal relevance for weather derivatives, in order to compare our performance to that of a very sophisticated leading meteorological forecast. One naturally suspects that the much larger information set on which the meteorological forecast is based will result in superior short-horizon point forecasting performance, but even if so, of great interest is the question of how quickly and with what pattern the superiority of the meteorological forecast deteriorates with forecast horizon.

We then progress to assess the performance of our model's long-horizon density forecasts, which are of maximal interest in weather derivative contexts, given the underlying option pricing considerations. Simultaneously, we also move to forecasting $H D D_{t}$ rather than $T_{t}$. This lets us match the most common weather derivative "underlying," and it also lets us explore the effects of using a daily model to produce much longer-horizon density forecasts.

\section{Point Forecasting}

We assess the short-term accuracy of daily average temperature forecasts based on our seasonal+trend+cycle model. In what follows, we refer to those forecasts as "autoregressive forecasts," for obvious reasons. We evaluate the autoregressive forecasts relative to three benchmark competitors, which range from rather naive to very sophisticated. The first benchmark forecast is a no-change forecast. The no-change forecast, often called the "persistence forecast" in the climatological literature, is the minimum mean squared error forecast at all horizons if daily average temperature follows a random walk.

The second benchmark forecast is from a more sophisticated two-component (seasonal+trend)

model. It captures (daily) seasonal effects via day-of-year dummy variables, in keeping with the common climatological use of daily averages as benchmarks, and it captures trend via a simple linear deterministic function of time. We refer to this forecast as the "climatological forecast." 
The third benchmark forecast, unlike benchmarks one and two, is not at all naive; on the contrary, it is a highly sophisticated forecast produced in real time by EarthSat. To produce their forecast, EarthSat meteorologists pool their expert judgement with model-based numerical weather prediction (NWP) forecasts from the National Weather Service, as well as forecasts from European, Canadian, and U.S. Navy weather services. This blending of judgement with models is typical of best-practice modern weather forecasting.

We were able to purchase approximately two years of forecasts from EarthSat. The sample period runs from 10/11/99, the date when EarthSat began to archive their forecasts electronically and make them publically available, through 10/22/01. Each weekday, EarthSat makes a set of $h$-day ahead daily average temperature forecasts, for $h=1,2, \ldots, 11$. EarthSat does not make forecasts on weekends.

We measure accuracy of all point forecasts using $h$-day-ahead root mean squared prediction error (RMSPE). We assess point forecasting accuracy at horizons of $h=1,2, \ldots, 11$ days, because those are the horizons at which EarthSat's forecasts are available. We compute measures of the accuracy of our model and the EarthSat model relative to that of the persistence and climatological benchmarks. RMPSE ratios relative to benchmarks are called skill scores in the meteorological literature (Brier and Allen, 1951) and U-statistics in the econometrics literature (Theil, 1966). Specifically, in an obvious notation, the skill score relative to the persistence forecast is $S k i l l_{h}^{p}=\sqrt{\sum\left(\hat{T}_{t+h, t}-T_{t+h}\right)^{2} / \sum\left(T_{t+h, t}^{p}-T_{t+h}\right)^{2}}$, where $T_{t+h, t}^{p}=T_{t}$ is the persistence forecast and $\hat{T}_{t+h, t}$ is either the autoregressive forecast or the EarthSat forecast. The skill score relative to the climatological forecast is $S k i l l_{h}^{c}=\sqrt{\sum\left(\hat{T}_{t+h, t}-T_{t+h}\right)^{2} / \sum\left(\hat{T}_{t+h, t}^{c}-T_{t+h}\right)^{2}}$, where $\hat{T}_{t+h, t}^{c}$ denotes the climatological forecast, $\hat{T}_{t+h, t}^{c}=\hat{\beta}_{0}+\hat{\beta}_{1}(t+h)+\sum_{i=1}^{365} \hat{\delta}_{i} D_{i, t+h}$, and $D_{i t}$ is a daily dummy.

A number of nuances merit discussion. First, for each of our time-series models, we estimate and forecast recursively, using only the data that were available in real time. Thus our forecasts at any time utilize no more average temperature information than do EarthSat's. In fact, our forecasts are based on less average temperature information: our forecast for day $t+1$ made on day $t$ is based on daily average 
temperature through 11:59 PM of day $t$, whereas the EarthSat forecast for day $t+1$, which is not released until 6:45 AM on day $t+1$, potentially makes use of the history of temperature through 6:45 AM of day $t+1$. Second, we make forecasts using our models only on the dates that EarthSat made forecasts. In particular, we make no forecasts on weekends. Hence, our accuracy comparisons proceed by averaging squared errors over precisely the same days as those corresponding to the EarthSat errors. This ensures a fair apples-to-apples comparison.

We report RMSPEs in Table 1 at horizons of $h=1,3,5,7,9$, and 11 days, for all cities and forecasting models. In addition, we graph skill scores as a function of horizon, against the persistence forecast in Figure 11 and against the climatological forecast in Figure 12, for all cities and horizons. The results are the same for all cities, so it is not necessary to discuss them individually by city. The results most definitely do differ, however, across models and horizons, as we now discuss. We first discuss the performance of the time-series forecasts, and then we discuss the EarthSat forecasts.

Let us consider first the forecasting performance of the persistence, climatological, and autoregressive models across the various horizons. First consider the comparative performance of the persistence and climatological forecasts. When $h=1$, the climatological forecasts are much worse than the persistence forecasts, reflecting the fact that persistence in daily average temperature renders the persistence forecast quite accurate at very short horizons. As the horizon lengthens, however, this result is reversed: the persistence forecast becomes comparatively poor, as the temperature today has rather little to with the temperature, for example, nine days from now.

Second, consider the performance of the autoregressive forecasts relative to the persistence and climatological forecasts. Even when $h=1$, the autoregressive forecasts consistently outperform the persistence forecast, and their relative superiority increases with horizon. The autoregressive forecasts also outperform the climatological forecasts at short horizons, but their comparative superiority decreases with horizon. The performance of the autoregressive forecast is commensurate with that of the climatological 
forecast roughly by the time $h=4$, indicating that the cyclical dynamics captured by the autoregressive model, which are responsible for its superior performance at shorter horizons, are not very persistent and therefore not readily exploited for superior forecast performance at longer horizons.

Now let us compare the forecasting performance of the autoregressive model and the EarthSat model. When $h=1$, the EarthSat forecasts are much better than the autoregressive forecasts (which in turn are better then either the persistence or climatological forecasts). Figures 11 and 12 make clear, however, that the EarthSat forecasts outperform the autoregressive forecasts by progressively less as the horizon lengthens, with nearly identical performance obtaining by the time $h=8$. One could even make a case that the point forecasting performances of EarthSat and our three-component model become indistinguishable before $h=8$ (say, by $h=5$ ) if one were to account for the sampling error in the estimated RMSPEs and for the fact that the EarthSat information set for any day $t$ actually contains a few hours of the next day.

All told, we view the point forecasting results as encouraging. At short horizons, time-series models produce forecasts at least as accurate, and often much more accurate, than those of standard benchmark persistence and climatological competitors, if nevertheless less accurate than state-of-the-art meteorological forecasts. At the longer horizons of relevance for weather derivatives, time-series models produce forecasts at least as accurate as those of competitors. One might assert, of course, that doing no worse than competitors at long horizons is faint praise, as all point forecasts revert fairly quickly to the climatological forecast, and hence all long-horizon forecasts are simply "equally poor." But such an assertion misses a crucial point: modern time-series methods can capture movements in conditional moments beyond the conditional mean, such as the conditional variance dynamics emphasized earlier, which feed into the density forecasts of ultimate relevance for weather derivatives, whose performance we now assess.

\section{Density Forecasting}

In this section, we shift our focus to long-horizon density forecasting, and to cumulative heating 
degree days, all of which is of crucial relevance for weather derivatives. Heating degree days for day $t$ is simply $H D D_{t}=\max \left(0,65-T_{t}\right)$. We use our model of daily average temperature to produce density forecasts of cumulative $H D D$ s from November 1 through March 31, for each city and for each year between 1960 and 2000, defined as $C u m H D D_{y, i}=\sum_{t=1}^{151} H D D_{t, y, i}$, for $y=1960, \ldots, 2000, i=1, \ldots, 4$. We remove February 29 from each leap year, so that each year is exactly 365 days long; hence each sum contains exactly 151 days. We use full-sample as opposed to recursive parameter estimates, as required by the very small number of CumHDD observations. To avoid unnecessarily burdensome notation, we will often drop the $y$ and $i$ subscripts when the meaning is clear from context.

We focus on CumHDD for two important reasons. First, weather derivative contracts are often written on the cumulative sum of a weather related outcome over a fixed horizon, as with the cumulative $H D D$ and $C D D$ contracts traded on the CME. Second, and related, the November-March $H D D$ contract is one of the most actively traded weather-related contracts and hence is of substantial direct interest.

On October 31 of each year, and for each city, we use the estimated daily model to produce a density forecast of CumHDD for the following winter's heating season. We simulate 250 realizations of CumHDD, which we then use to estimate the density, as follows. First, we simulate 250 151-day realizations of the temperature shock $\varepsilon_{t}$, by drawing with replacement from the empirical distribution of estimated temperature shocks $\left(\hat{\boldsymbol{\varepsilon}}_{t}\right)$. Second, we run the 250 151-day realizations of temperature shocks through the estimated model (1) to obtain 250 simulated 151-day realizations of daily average temperature. Third, we convert the 250 simulated 151-day realizations of daily average temperature into 250 simulated 151-day realizations of $H D D$, which we cumulate over the November-March heating season, CumHDD $_{s}=\sum_{t=1}^{151} H D D_{t, s}, s=1,2, \ldots, 250$. Finally, we form the empirical distribution function of CumHDD, based upon CumHDD $_{s}, s=1, \ldots, 250$.

After passing through the entire sample, we have 41 assessed distribution functions, $\hat{F}_{y}(\bullet)$, $y=1960, \ldots, 2000$, one governing each of $C u m H D D_{y}, y=1960, \ldots ., 2000$. We assess the conditional $-13-$ 
calibration of those distributional forecasts via the probability integral transform, as suggested and extended by Rosenblatt (1952) and extended by Dawid (1984), Diebold, Gunther and Tay (1998), and Diebold, Hahn and Tay (1999). In particular, if the estimated distribution and true distribution coincide year-by-year, then the series of assessed distribution functions $\hat{F}_{y}(\bullet)$ evaluated at the corresponding series of realized values of $C u m H D D_{y}$ should be approximately iid and uniformly distributed on the unit interval. Formally, $z_{y} \equiv \hat{F}_{y}\left(C u m H D D_{y}\right) \stackrel{\text { iid }}{\sim} U(0,1)$. For each city, we check uniformity by examining histograms of $z$, and we check independence by examining correlograms of the first four powers of $z$. The sample of size 41 is of course small, but the framework has previously been applied successfully in small samples, as for example by Diebold, Tay and Wallis (1999).

First consider assessing uniformity. We estimate the density of $z$ using simple four-bin histograms, which we present in the leftmost column of Figure 13, accompanied by $95 \%$ pointwise error bands under the iid $\mathrm{U}(0,1)$ null hypothesis. Interestingly, the $z$ series differ rather noticeably from uniformity, and moreover, they display a common pattern: too many large CumHDD realizations occur relative to the assessed distributions, as evidenced by the increase in the histograms when moving from left to right. The common nature of uniformity violations may indicate a neglected common temperature component, due for example to El Niño, La Niña, changes in the jet stream, or various other global factors.

Now consider assessing independence. In the last four columns of Figure 13, we show the correlograms of the first four powers of $z$, taken to a maximum displacement of ten years, together with asymptotic $95 \%$ Bartlett bands under the iid null hypothesis. The results are mixed, but a common pattern of some positive serial correlation is often apparent.

All told, although our CumHDD distributional forecasting performance is encouraging in certain respects, there is nevertheless clear room for improvement. Evidently the effects of small specification errors in the daily model, which have negligible consequences for near-term forecasting, cumulate as the horizon lengthens, producing large consequences for longer-term forecasting. This makes perfect sense 
when one considers that the error in forecasting CumHDD is of course the sum of the many component daily errors, and that the variance of a sum is the sum of the variances plus the sum of all possible pairwise covariances. Hence tiny and hard-to-detect but slowly-decaying serial correlation in 1-day-ahead daily average temperature forecasting errors may cumulate over long horizons.

In future work beyond the scope of the present paper, it will be of interest to model and forecast CumHDD directly. Presently we fit only a single (daily) average temperature model, which we estimate by minimizing a loss function corresponding to 1-day-ahead mean squared prediction error, and we then use the model to produce forecasts at many different horizons, all of which feed into our CumHDD forecasts.

\section{Concluding Remarks and Directions for Future Research}

Weather modeling and forecasting are crucial to both the demand and supply sides of the weather derivatives market. On the demand side, to assess the potential for hedging against weather surprises and to formulate the appropriate hedging strategies, one needs to determine how much "weather noise" exists for weather derivatives to eliminate, and that requires weather modeling and forecasting. On the supply side, standard approaches to arbitrage-free pricing are irrelevant in weather derivative contexts, and so the only way to price options reliably is again by modeling and forecasting the underlying weather variable.

Rather curiously, it seems that little thought has been given to the crucial question of how best to approach weather modeling and forecasting in the context of weather derivative demand and supply. The vast majority of extant weather forecasting literature has a structural "atmospheric science" feel, and although such an approach may well be best for forecasting twelve hours ahead, as verified both by our own results and those of numerous others, it is not obvious that it is best for the longer horizons relevant for weather derivatives, such as twelve weeks or six months. Moreover, it is distributional forecasts, not point forecasts, that are of maximal relevance in the derivatives context. Good distributional forecasting does not necessarily require a structural model, but it does require accurate approximations to stochastic dynamics.

In this paper we took an arguably-naive nonstructural time-series approach to modeling and 
forecasting daily average temperature in four U.S. cities, and we inquired systematically as to whether it proves useful. The answer, perhaps surprisingly, is a qualified yes. Time-series modeling reveals a wealth of information about both conditional mean dynamics and conditional variance dynamics of daily average temperature, and it provides insights into both the distributions of temperature and temperature surprises, and the differences between them. The success of time-series modeling in capturing conditional mean dynamics translates into successful point forecasting. Our point forecasts dominated the persistence and climatological forecasts, but were still not as good as the judgementally-adjusted NWP forecast produced by EarthSat until a horizon of eight days, after which all point forecasts perform equally well.

We routinely found strong seasonality in weather surprise volatility, and we assessed the adequacy of long-horizon distributional forecasts that accounted for it, with mixed but encouraging results. Interestingly, we found noticeable commonality in the patterns of cross-city deviations from perfect conditional calibration, indicating possible dependence on common latent components, perhaps due to El Niño or La Niña.

All told, we would assert that in the context of weather modeling as relevant for weather derivatives, it appears that the performance of simple yet sophisticated time-series models and forecasts is at least good enough to suggest the desirability of additional exploration. When, in addition, one considers that time-series models and methods are inexpensive, easily replicated, easily extended, beneficially intrinsically stochastic, and capable of producing both point and density forecasts at a variety of horizons, we feel that a strong case exists for their use in the context of modeling and forecasting as relevant for weather derivatives.

We would also assert that our views are consistent with the mainstream consensus in atmospheric science. In his well-known text, for example, Wilks (1995, p. 159) notes that "[Statistical weather forecasting] methods are still viable and useful at very short lead times (hours in advance) or very long lead times (weeks or more in advance) for which NWP information is either not available with sufficient 
promptness or accuracy, respectively." Indeed, in many respects our results are simply an extensive confirmation of Wilks' assertion in the context of weather derivatives, which are of great current interest.

Ultimately, our view on weather forecasting for weather derivatives has evolved toward recognition that climatological forecasts are what we need, but that standard point climatological forecasts - effectively little more than daily averages - are much too restrictive. Instead, we seek "generalized climatological forecasts" from richer models tracking entire conditional distributions, and time-series methods may have much to contribute. Our model quantifying the time-varying conditional variance of daily average temperature is one step toward a fully generalized climatological forecast, but many issues remain unexplored. Here we briefly discuss a few that we find particularly intriguing.

Arguably our central modeling innovation centers on the dynamics of the conditional variance. But richer dynamics could also be beneficially permitted in both lower-ordered conditional moments (the conditional mean) and higher-ordered conditional moments (such as the conditional skewness and kurtosis). As regards the conditional mean, one could introduce explanatory variables, as in Visser and Molenaar (1995), who condition on a volcanic activity index, sunspot numbers, and a southern oscillation index. Relevant work also includes Jones (1996) and Pozo et al. (1998), but all of those papers use annual data and therefore miss the seasonal patterns in both conditional mean and conditional variance dynamics so crucial for weather derivatives demand and supply. One could also allow for stochastic regime switching, which might aid, for example, in the detection of El Niño and La Niña events. (See Richman and Montroy, 1996, and also Zwiers and von Storch, 1990.) Note that allowance for stochastic regime switching is very different from structural break detection, caused, for example, by a change in measurement location, measurement equipment, observation time. As regards the conditional skewness and kurtosis, one could model them directly, as for example with the autoregressive conditional skewness model of Harvey and Siddique (1999). Alternatively, one could directly model the evolution of the entire conditional density, as in Hansen (1994). 
Aspects of multivariate analysis and cross-hedging also hold promise for future work. Cross-city correlations may be crucially important, because they govern the potential for cross-hedging. Hedging weather risk in a remote Midwestern location might, for example, be prohibitively expensive or even impossible due to illiquid or nonexistent markets, but if that risk is highly correlated with Chicago's weather risk, for which a liquid market exists, effective hedging may still be possible. An obvious and important extension of the univariate temperature analysis reported in the present paper is vector autoregressive modeling of daily average temperature in a set of cities, allowing for a time-varying innovation variance-covariance matrix. Of particular interest would be the fitted and forecasted conditional mean, variance and covariance dynamics, the covariance matrices of standardized innovations, and the impulse response functions (which chart the speed and pattern with which weather surprises in one location are transmitted to other locations).

Another interesting multivariate issue involves weather-related swings in earnings and share prices. One might conjecture that once Wall Street recognizes that there are effective ways to manage weather risk, weather-related swings in earnings will no longer be tolerated. It will be of interest to use the size of weather-related swings in earnings as way to assess the potential for weather derivatives use. In particular, we need to understand how weather surprises translate into earnings surprises, which then translate into stock return movements. Some interesting subtleties may arise. As one example, note that only systematic weather risk should be priced, which raises the issue of how to disentangle systematic and non-systematic weather risks. As a second example, note that there may be nonlinearities in the relationship between prices and the weather induced via path dependence; if there is a freeze early-on, for example, then it doesn't matter how good the weather is subsequently: the crop will be ruined and prices will be high (see Richardson, Bodoukh, Sjen, and Whitelaw, 2001).

\section{References}

Bloomfield, P. (1992), “Trends in Global Temperature," Climate Change, 21, 1-16. 
Bollerslev, T. and Wooldridge, J.M. (1992), “Quasi-Maximum Likelihood Estimation and Inference in Dynamic Models with Time Varying Covariances," Econometric Reviews, 11, 143-172.

Brier, G.W. and Allen, R.A. (1951), "Verification of Weather Forecasts," in T.F. Malone (ed.), Compendium of Meteorology, American Meteorological Society.

Campbell, S.D. and Diebold, F.X. (2002), “Weather Forecasting for Weather Derivatives,” Working Paper No. 02-42, Wharton Financial Institutions Center.

Cao, M. and Wei, J. (2001), "Pricing Weather Derivatives: An Equilibrium Approach," Manuscript, University of York and University of Toronto.

Davis, M. (2001), "Pricing Weather Derivatives by Marginal Value," Quantitative Finance, 1, 305-308.

Dawid, A.P. (1984), "Statistical Theory: The Prequential Approach," Journal of the Royal Statistical Society, Series A, 147, 278-292.

Diebold, F.X. (2004), Elements of Forecasting, Third Edition. Cincinnati: South-Western College Publishing.

Diebold, F.X., Gunther, T. and Tay, A.S. (1998), "Evaluating Density Forecasts, with Applications to Financial Risk Management," International Economic Review, 39, 863-883.

Diebold, F.X., Hahn, J. and Tay, A.S. (1999), "Multivariate Density Forecast Evaluation and Calibration in Financial Risk Management: High-Frequency Returns on Foreign Exchange,” Review of Economics and Statistics, 81, 661-673.

Diebold, F.X., Tay, A.S. and Wallis, K. (1999), “Evaluating Density Forecasts of Inflation: The Survey of Professional Forecasters," in R. Engle and H. White (eds.), Cointegration, Causality, and Forecasting: A Festschrift in Honor of Clive W.J. Granger, 76-90, Oxford University Press.

Engle, R.F. (1982), “Autoregressive Conditional Heteroskedasticity with Estimates of the Variance of U.K. Inflation," Econometrica, 50, 987-1008.

Geman, H., Ed. (1999), Insurance and Weather Derivatives: From Exotic Options to Exotic Underlyings. 
London: Risk Publications.

Hansen, B.E. (1994), “Autoregressive Conditional Density Estimation,” International Economic Review, 35, 705-730.

Harvey, A.C. (1989), Forecasting, Structural Time Series Models and the Kalman Filter. Cambridge: Cambridge University Press.

Harvey, C.R. and Siddique, A. (1999), “Autoregressive Conditional Skewness," Journal of Financial and Quantitative Analysis, 34, 465-488.

Hyndman, R.J. and Grunwald, G.K. (2000), “Generalized Additive Modeling of Mixed Distribution Markov Models with Application to Melbourne's Rainfall," Australian and New Zealand Journal of Statistics, 42, 145-158.

Jones, R.H. (1996), “The Potential of State Space Models in Statistical Meteorology,” Proceedings of the $13^{\text {th }}$ Conference on Probability and Statistics in the Atmospheric Sciences, American Meteorological Society.

Milionis, A.E. and Davies, T.D. (1994), "Box-Jenkins univariate Modeling for Climatological Time Series Analysis: An Application to the Monthly Activity of Temperature Inversions," International Journal of Climatology, 14 569-579.

Neese, J.M. (1994), "Systematic Biases in Manual Observations of Maximum and Minimum Temperature," Journal of Climate, 7, 834-842.

Nelson, D.B. (1991), "Conditional Heteroskedasticity in Asset Returns: A New Approach," Econometrica, $59,347-370$.

Pozo, D., Esteban-Parra, M.J., Rodrigo, F.S. and Castro-Diez, Y. (1998), “ARIMA Structural Models for Temperature Time Series of Spain," Proceedings of the $14^{\text {th }}$ Conference on Probability and Statistics in the Atmospheric Sciences, American Meteorological Society.

Richardson, M., Bodoukh, J., Sjen, Y. and Whitelaw, R. (2001), "Freshly Squeezed: A Reinterpretation of $-20-$ 
Market Rationality in the Orange Juice Futures Market," Working Paper, New York University.

Richman, M.B. and Montroy, D.L. (1996), "Nonlinearities in the Signal Between El Niño / La Niña Events and North American Precipitation and Temperature," Proceedings of the $13^{\text {th }}$ Conference on Probability and Statistics in the Atmospheric Sciences, American Meteorological Society.

Roll, R. (1984), “Orange Juice and Weather,” American Economic Review, 74, 861-880.

Rosenblatt, M. (1952), "Remarks on a Multivariate Transformation," Annals of Mathematical Statistics, 23, $470-472$.

Seater, J.J. (1993), "World Temperature-Trend Uncertainties and Their Implications for Economic Policy," Journal of Business and Economic Statistics, 11, 265-277.

Storch, H. von and Zwiers, F.W. (1999), Statistical Analysis in Climate Research. Cambridge: Cambridge University Press.

Theil, H. (1966), Applied Economic Forecasting. Amsterdam: North-Holland.

Torro, H., Meneu, V. and Valor E. (2001), "Single Factor Stochastic Models with Seasonality Applied to Underlying Weather Derivatives Variables,” Manuscript, University of Valencia.

Tribia, J.J. (1997), "Weather Prediction," in R. Katz and A. Murphy (eds.), Economic Value of Weather and Climate Forecasts. Cambridge: Cambridge University Press.

Visser, H. and Molenaar, J. (1995), “Trend Estimation and Regression Analysis in Climatological Time Series: An Application of Structural Time Series Models and the Kalman Filter," Journal of Climate, 8, 969-979.

Weather Risk (1998), Supplement to Risk Magazine, October.

Weather Risk (2000), Supplement to Risk Magazine, August.

Wilks, D.S. (1995), Statistical Methods in the Atmospheric Sciences. New York: Academic Press.

Zwiers, F.W. and von Storch, H. (1990), "Regime Dependent Autoregressive Time Series Modeling of the Southern Oscillation," Journal of Climate, 3, 1347-1363. 
Table 1

Point Forecast Accuracy Comparisons

Daily Average Temperature

\begin{tabular}{|c|c|c|c|c|c|c|}
\hline & 1-Day Ahead & 3-Day-Ahead & 5-Day-Ahead & 7-Day-Ahead & 9-Day-Ahead & 11-Day-Ahead \\
\hline \multicolumn{7}{|l|}{ Atlanta } \\
\hline Persistence & 4.50 & 8.00 & 8.72 & 9.07 & 8.99 & 9.28 \\
\hline Climatological & 6.93 & 6.88 & 6.84 & 7.04 & 6.93 & 6.59 \\
\hline Autoregressive & 4.12 & 6.45 & 6.69 & 7.03 & 6.89 & 6.59 \\
\hline EarthSat & 2.74 & 3.84 & 5.10 & 6.04 & 6.65 & 7.00 \\
\hline \multicolumn{7}{|l|}{ Chicago } \\
\hline Persistence & 6.73 & 10.50 & 11.06 & 11.54 & 11.74 & 11.99 \\
\hline Climatological & 8.74 & 8.72 & 8.72 & 8.50 & 8.88 & 8.55 \\
\hline Autoregressive & 6.06 & 8.38 & 8.57 & 8.45 & 8.84 & 8.53 \\
\hline EarthSat & 3.22 & 4.70 & 6.31 & 7.46 & 8.48 & 8.92 \\
\hline \multicolumn{7}{|l|}{$\underline{\text { Las Vegas }}$} \\
\hline Persistence & 3.78 & 6.15 & 7.08 & 7.71 & 7.96 & 7.93 \\
\hline Climatological & 5.99 & 5.85 & 5.80 & 6.02 & 5.97 & 5.80 \\
\hline Autoregressive & 3.57 & 5.20 & 5.58 & 5.92 & 5.97 & 5.78 \\
\hline EarthSat & 2.54 & 3.28 & 4.19 & 5.32 & 5.81 & 6.04 \\
\hline \multicolumn{7}{|l|}{ Philadelphia } \\
\hline Persistence & 5.53 & 8.83 & 9.83 & 9.87 & 9.55 & 10.18 \\
\hline Climatological & 7.12 & 6.95 & 7.27 & 7.19 & 6.98 & 7.19 \\
\hline Autoregressive & 4.95 & 6.74 & 7.23 & 7.15 & 6.95 & 7.08 \\
\hline EarthSat & 2.61 & 3.91 & 5.35 & 6.26 & 7.24 & 8.37 \\
\hline
\end{tabular}


Figure 1: Time-Series Plots of Daily Average Temperature
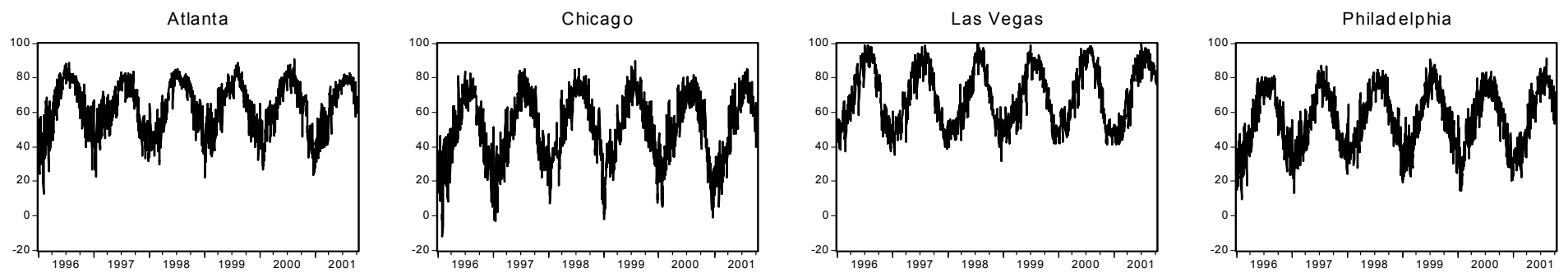

Figure 2: Estimated Unconditional Distributions of Daily Average Temperature
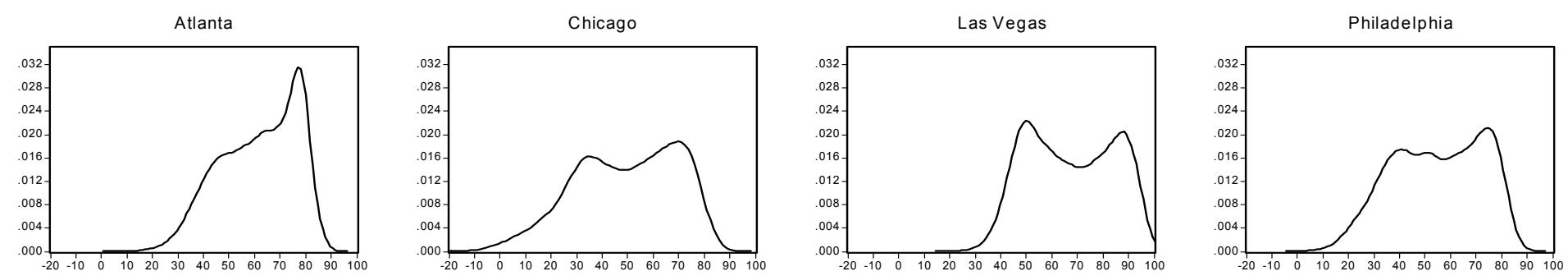

Figure 3: Estimated Seasonal Patterns, Daily Average Temperature
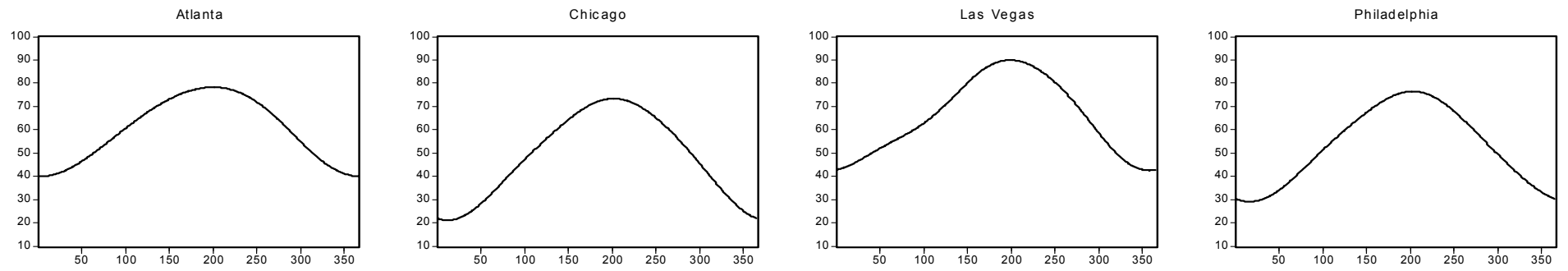

Figure 4: Estimated Model Residuals, Daily Average Temperature
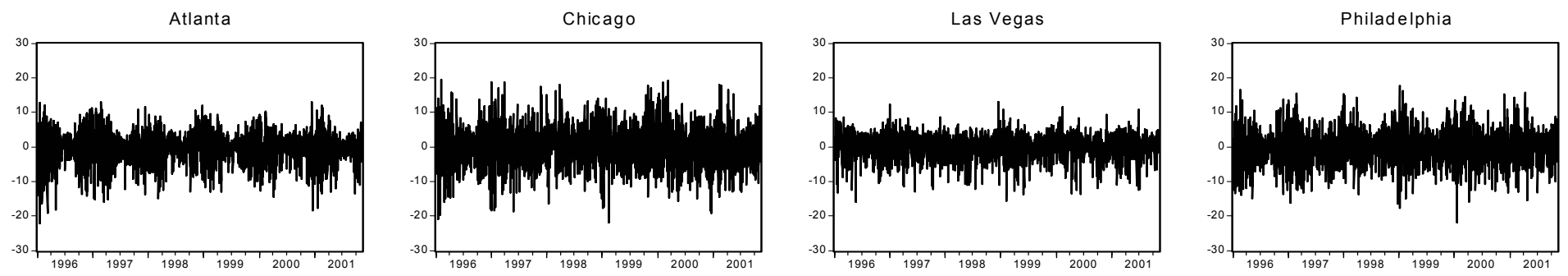
Figure 5: Estimated Unconditional Distribution of Residuals, Daily Average Temperature
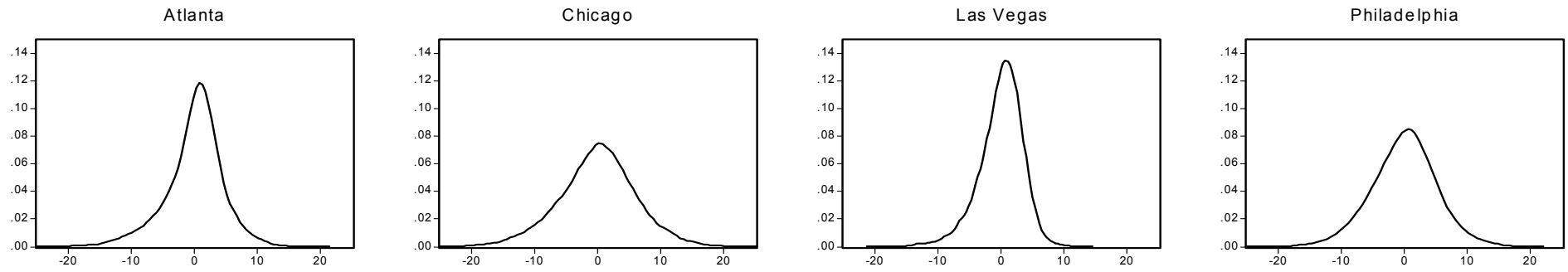

Figure 6: Correlogram of Residuals, Daily Average Temperature
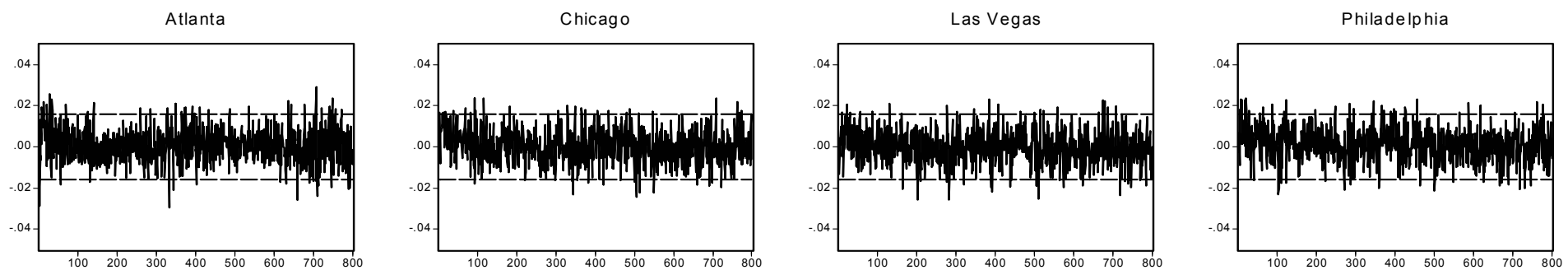

Figure 7: Correlogram of Squared Residuals, Daily Average Temperature
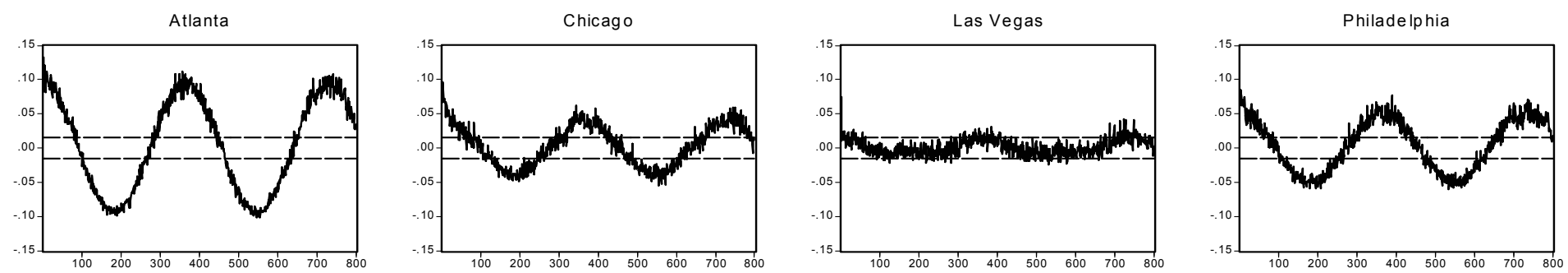

Figure 8: Estimated Conditional Standard Deviations, Daily Average Temperature

Atlanta

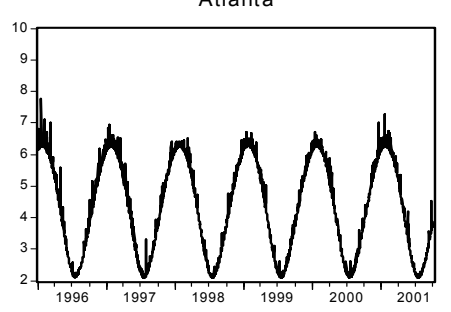

Chicago

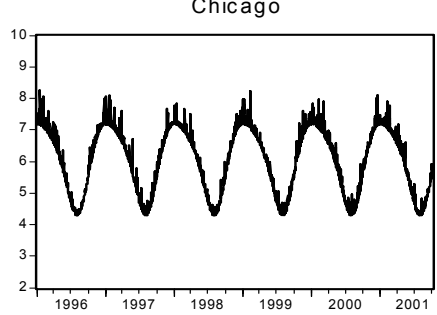

Las Vegas

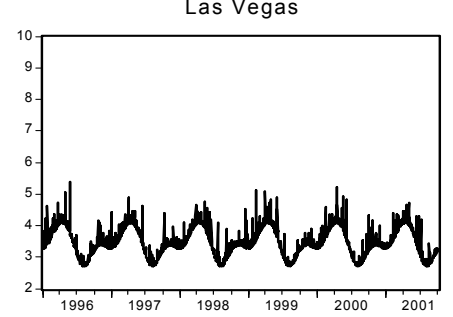

Philadelphia

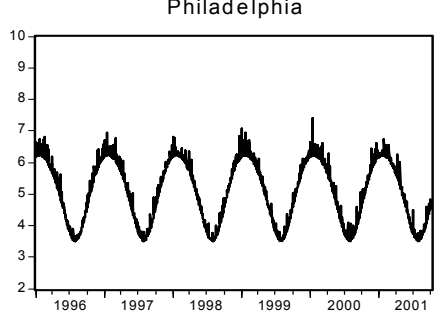


Figure 9: Estimated Unconditional Distribution of Standardized Residuals, Daily Average Temperature
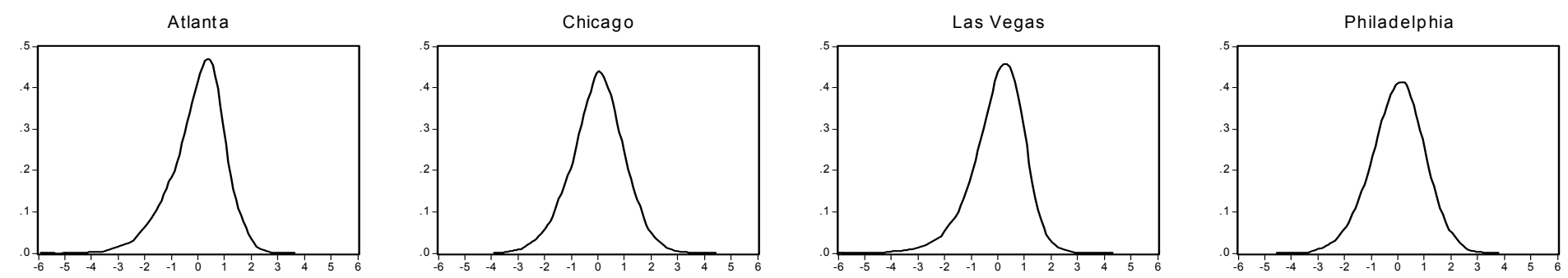

Figure 10: Correlograms of Squared Standardized Residuals, Daily Average Temperature
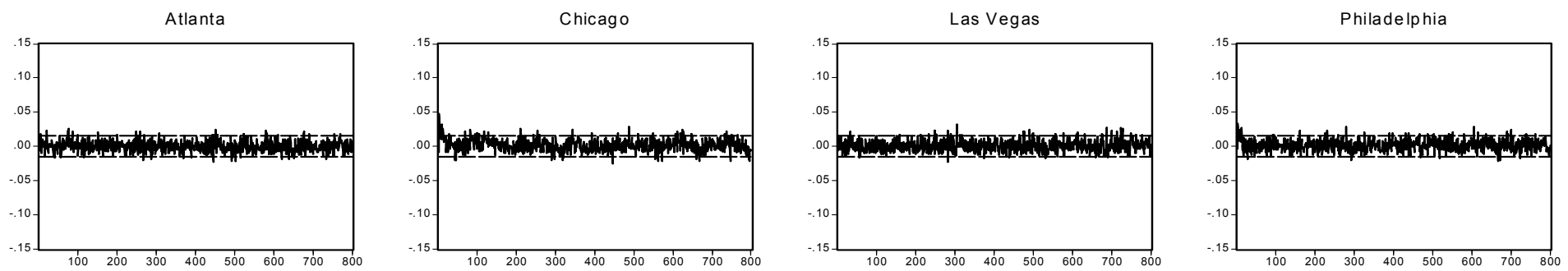

Figure 11: Forecast Skill Relative to Persistence Forecasts, Daily Average Temperature Point Forecasts
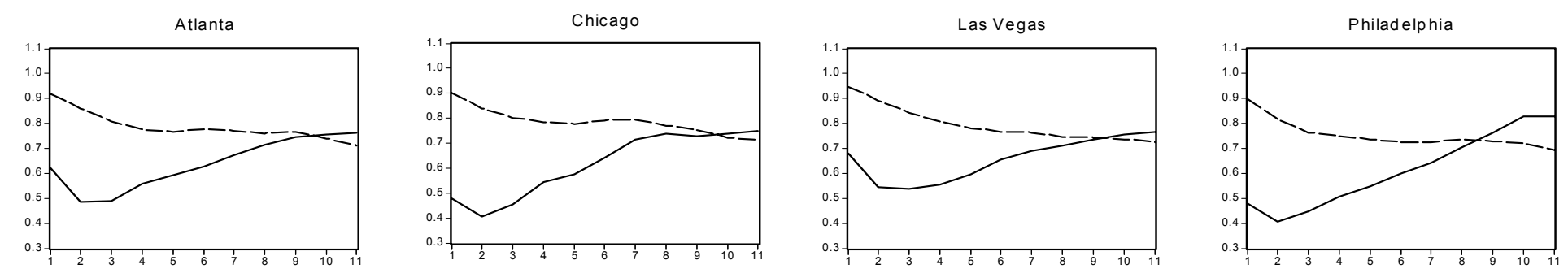

Figure 12: Forecast Relative to Climatological Forecast, Daily Average Temperature
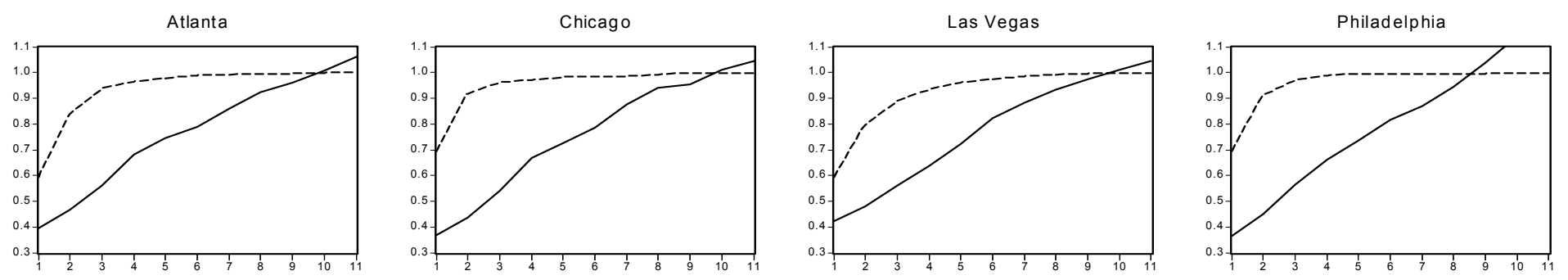
Figure 13: z-Statistics Distributions and Dynamics, Daily Average Temperature Distributional Forecasts
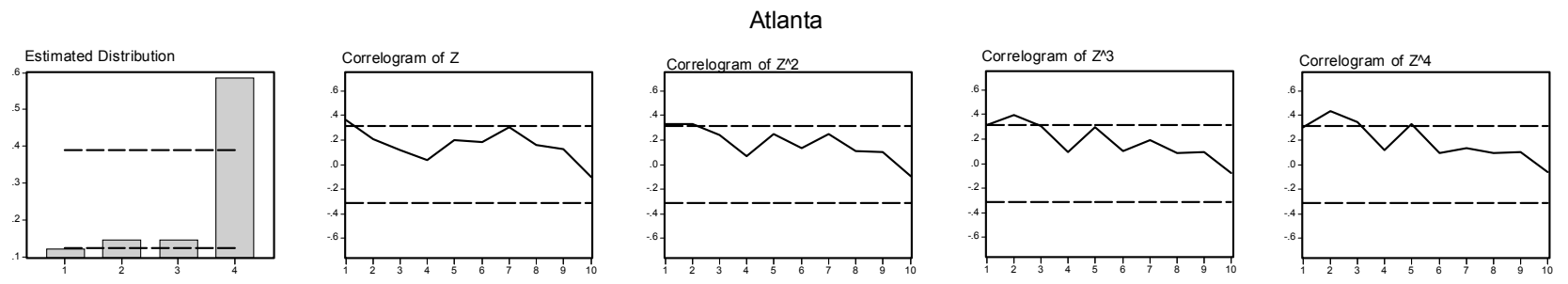

Chicago
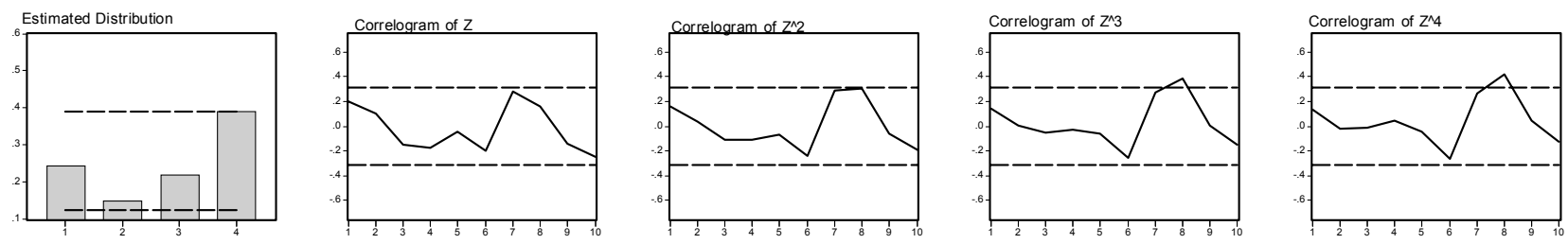

Las Vegas
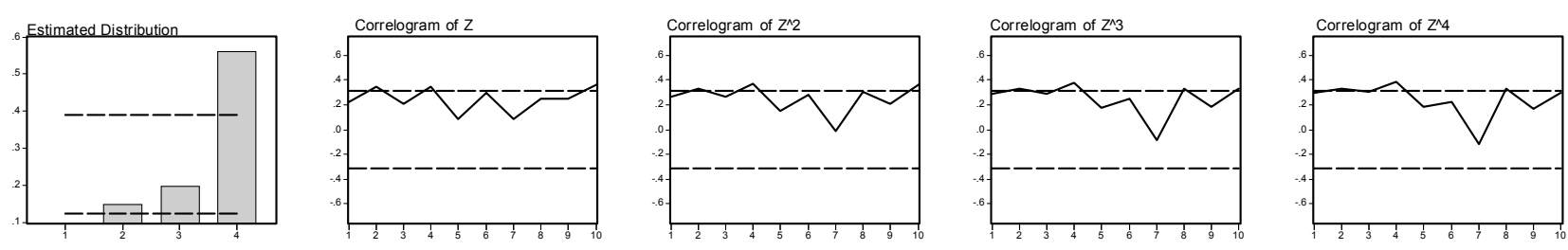

Philadelphia
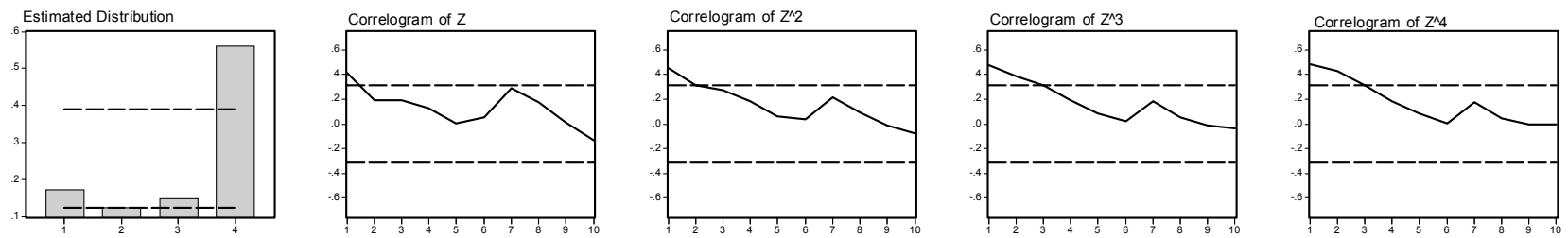


\section{Notes to Tables and Figures}

Notes to Table 1: We show each forecast's root mean squared error, measured in degrees Fahrenheit.

Notes to Figure 1: Each panel displays a time-series plot of daily average temperature, 1996-2001.

Notes to Figure 2: Each panel displays a kernel density estimate of the unconditional distribution of daily average temperature, 1960-2001. In each case, we employ the Epanechnikov kernel and select the bandwidth using Silverman's rule, $h=0.9 \hat{\sigma} N^{-0.2}$.

Notes to Figure 3: We show the seasonal patterns estimated from Fourier models, Seasonal $_{t}=\sum_{p=1}^{3}\left(\sin \left(2 \pi p \frac{n(t)}{365}\right)+\beta_{i} \cos \left(2 \pi p \frac{n(t)}{365}\right)\right)$, where we approximate daily average temperature dynamics by the unobserved-components model, $T_{t}=$ Trend $_{t}+$ Seasonal $_{t}+\sum_{i=1}^{25} \rho_{i} T_{t-i}+\sigma_{t} \varepsilon_{t}$, as discussed in detail in the text.

Notes to Figure 4: Each panel displays the residuals from an unobserved-components model, $T_{t}=$ Trend $_{t}+$ Seasonal $_{t}+\sum_{i=1}^{25} \rho_{i} T_{t-i}+\sigma_{t} \varepsilon_{t}$, as discussed in detail in the text, 1996-2001.

Notes to Figure 5: Each panel displays a kernel density estimate of the distribution of the residuals from our daily average temperature model, $T_{t}-\hat{T r}_{r} \mathrm{nd}_{t}-\hat{S}_{\text {easonal }}-\sum_{i=1}^{25} \hat{\rho}_{i} T_{t-i}$, as discussed in detail in the text. In each case, we employ the Epanechnikov kernel and select the bandwidth using Silverman's rule, $h=0.9 \hat{\sigma} N^{-0.2}$.

Notes to Figure 6: Each panel displays sample autocorrelations of the residuals from our daily average temperature model, $T_{t}-\hat{T}_{r e n d}-\hat{S}_{t}$ easonal ${ }_{t}-\sum_{i=1}^{25} \hat{\rho}_{i} T_{t-i}$, together with Bartlett's approximate ninety-five percent confidence intervals under the null hypothesis of white noise, as discussed in detail in the text.

Notes to Figure 7: Each panel displays sample autocorrelations of the squared residuals from our daily average temperature model, $\left(T_{t}-\hat{T r}_{\text {rend }}-\hat{S}_{t} \text { easonal } t-\sum_{i=1}^{25} \hat{\rho}_{i} T_{t-i}\right)^{2}$, together with Bartlett's approximate ninety-five percent confidence intervals under the null hypothesis of white noise, as discussed in detail in the text. 
Notes to Figure 8: Each panel displays a time series of estimated conditional standard deviations $\left(\hat{\sigma}_{t}\right)$ of daily average temperature obtained from the model, where $\hat{\sigma}_{t}^{2}=\sum_{q=1}^{3}\left(\hat{\gamma}_{c, q} \cos \left(2 \pi q \frac{n(t)}{365}\right)+\hat{\gamma}_{s, q} \sin \left(2 \pi q \frac{n(t)}{365}\right)\right)+\hat{\alpha} \varepsilon_{t-1}^{2}+\hat{\beta} \hat{\sigma}_{t-1}^{2}, 1996-2001$.

Notes to Figure 9: Each panel displays a kernel density estimate of standardized residuals from our average temperature model, $\left(T_{t}-\hat{T r}_{\mathrm{r} e n d_{t}}-\hat{S}_{\text {easonal }}-\sum_{i=1}^{25} \hat{\rho}_{i} T_{t-i}\right) / \hat{\sigma}_{t}$. In each case, we employ the Epanechnikov kernel and select the bandwidth using Silverman's rule, $h=0.9 \hat{\sigma} N^{-0.2}$.

Notes to Figure 10: Each panel displays sample autocorrelations of the squared standardized residuals from our daily average temperature model, $\left(\left(T_{t}-\hat{T} \text { rend } t-\hat{S} \text { easonal } t-\sum_{i=1}^{25} \hat{\rho}_{i} T_{t-i}\right) / \hat{\sigma}_{t}\right)^{2}$, together with Bartlett's approximate ninety-five percent confidence intervals under the null hypothesis of white noise, as discussed in detail in the text.

Notes to Figure 11: Each panel displays the ratio of a forecast's RMSPE to that of a persistence forecast, for 1-day-ahead through 11-day-ahead horizons. The solid line refers to the EarthSat forecast, and the dashed line refers to the autoregressive forecast. The forecast evaluation period is $10 / 11 / 99-10 / 22 / 01$, as discussed in detail in the text.

Notes to Figure 12: Each panel displays the ratio of a forecast's RMSPE to that of a climatological forecast, for 1-day-ahead through 11-day-ahead horizons. The solid line refers to the EarthSat forecast, and the dashed line refers to the autoregressive forecast. The forecast evaluation period is 10/11/99 $10 / 22 / 01$, as discussed in detail in the text.

Notes to Figure 13: Each row displays a histogram for $z$, as well as correlograms for the first four powers of $z$, where $z$ is the probability integral transform of cumulative November-March $H D D$ s, 1960-2000. Dashed lines indicate approximate ninety-five percent confidence intervals in the iid $U(0,1)$ case corresponding to correct conditional calibration. See text for details. 